\title{
Chip-Level Interconnections Realized Via the Laser-Induced Forward Transfer Technique
}

\author{
K.S. Kaur, J. Missinne and G. Van Steenberge \\ Centre for Microsystems Technology, imec/Ghent University, Technologiepark 914A, B-9052 Gent, Belgium \\ email:Kaur.Kamalpreet@elis.ugent.be
}

\begin{abstract}
In this paper, successful flip-chip bonding and DC characterization of single photodiode and VCSEL chips via Laser-Induced Forward Transfer (LIFT) printed micro-bumps of indium, silver nano-particle (AgNP) based inks and pastes, is reported.

OCIS codes: (130.6622) Subsystem integration and techniques; (140.3390) Laser materials processing; (200.4650) Optical interconnects
\end{abstract}

\section{Introduction}

The key to meet an ever-increasing demand for ultra-miniaturized high-density circuits in the micro-electronics industry, is the use of efficient, cost-effective interconnection methods. Single chip-level bumping and bonding is critical for fabricating ultra-miniature circuitry. The existing interconnection techniques however, do not provide a combination of flexibility and high accuracy required especially for fine-pitch bonding on chip-scale. In this paper, we report the use of a laser-based bumping process called laser-induced forward transfer (LIFT) for flip-chip (FC) bonding of single photodiode (PD) and vertical-cavity surface-emitting laser (VCSEL) chips (schematic shown in fig. 1(a)). LIFT is an additive direct-write method for controlled patterning and printing (in a single-step) of a wide range of materials and devices with micron and sub-micron resolution [1-3]. In addition, LIFT offers several advantages such as low temperature processing, flexibility, simplicity and high accuracy, that makes it an excellent candidate to realize chip-level interconnections.

\section{Experiments and results}

\subsection{LIFT-assisted micro-bumping}

Three different bonding materials (the donor) namely: indium (In), silver nano-particle (AgNP) based ink (U5603 from Sunchemicals) and paste (PA-010 from InkTec) were used for the LIFT experiments. Thin films of these materials were deposited onto glass substrates (the carrier) employing evaporation (In), spin coating (AgNP ink, $3000 \mathrm{rpm}, 20 \mathrm{~s}$ ) and doctor blading (AgNP paste) techniques. After spin coating the AgNP ink samples were kept for $\sim 4$ hours under ambient conditions in order to achieve an optimum AgNP concentration and viscosity. For the case of indium, six bumps were stacked on top of each other sequentially to achieve a bump thickness of $1.5 \mu \mathrm{m}$. Glass substrates with $80 \mu \mathrm{m} \times 80 \mu \mathrm{m} \times 5 \mu \mathrm{m}$ lithographically patterned Ni-Au plated bond-pads were used as the receiver. The donor-receiver assembly was then mounted on a computer-controlled 2D translation stage. Single laser pulses from a Timebandwidth Duetto $(355 \mathrm{~nm}, 12 \mathrm{ps})$ were then focused at the carrier-donor interface thereby, enabling the forward-transfer of donor micro-dots onto the receiver bond pads. Figure 1 (a) and 1 (b) show the optical micrographs of typical bumped receiver substrates for a PD and VCSEL chip respectively. All the experiments were performed in air and at room temperature.

\subsection{Thermo-compression bonding of optoelectronic components}

The next step in the process was to bond optoelectronic components to the LIFT-assisted bumped receiver substrates. Commercially available VCSEL array chips $\left(1 \times 4,5 \mathrm{~Gb} / \mathrm{s}, 850 \mathrm{~nm}, 1 \times 0.350 \times 0.150 \mathrm{~mm}^{3}\right)$ from ULM photonics and PD array chips $\left(1 \times 4,10 \mathrm{~Gb} / \mathrm{s}, 850 \mathrm{~nm}, 1 \times 0.450 \times 0.15 \mathrm{~mm}^{3}\right)$ from Albis Optoelectronics were chosen as the optoelectronic components. A semi-automatic flip-chip bonder (T-320X) from Tresky was used to bond these VCSEL and PD chips to the receiver substrates and to establish an electrical and mechanical interconnection between them. The various key experimental parameters for LIFTing and bonding are depicted in the table below. Figure 2 shows flip-chip bonded (FCB) PD and VCSEL assemblies as observed under an optical microscope from the backside of the receiver glass substrates. To enhance the reliability of the bonded assemblies an 
optically transparent UV curable adhesive (NOA 86 from Norland) was used for encapsulation. The adhesive was dispensed around the edges of the bonded chips followed by curing $30 \mathrm{~s}$ using an Omnicure $1000 \mathrm{UV}$ lamp.

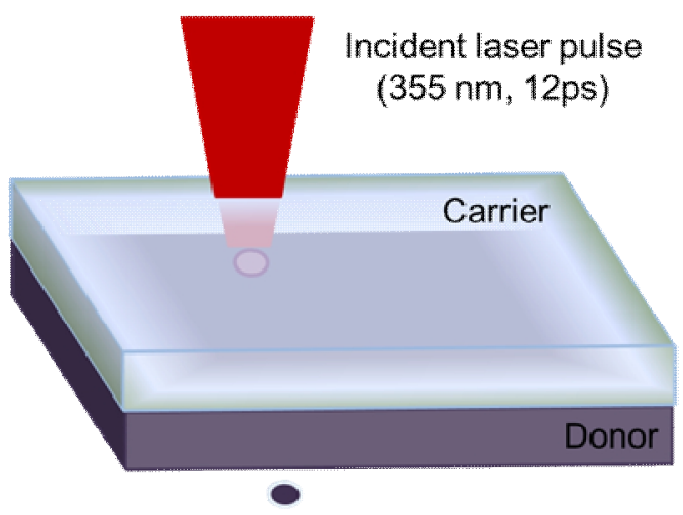

(a)
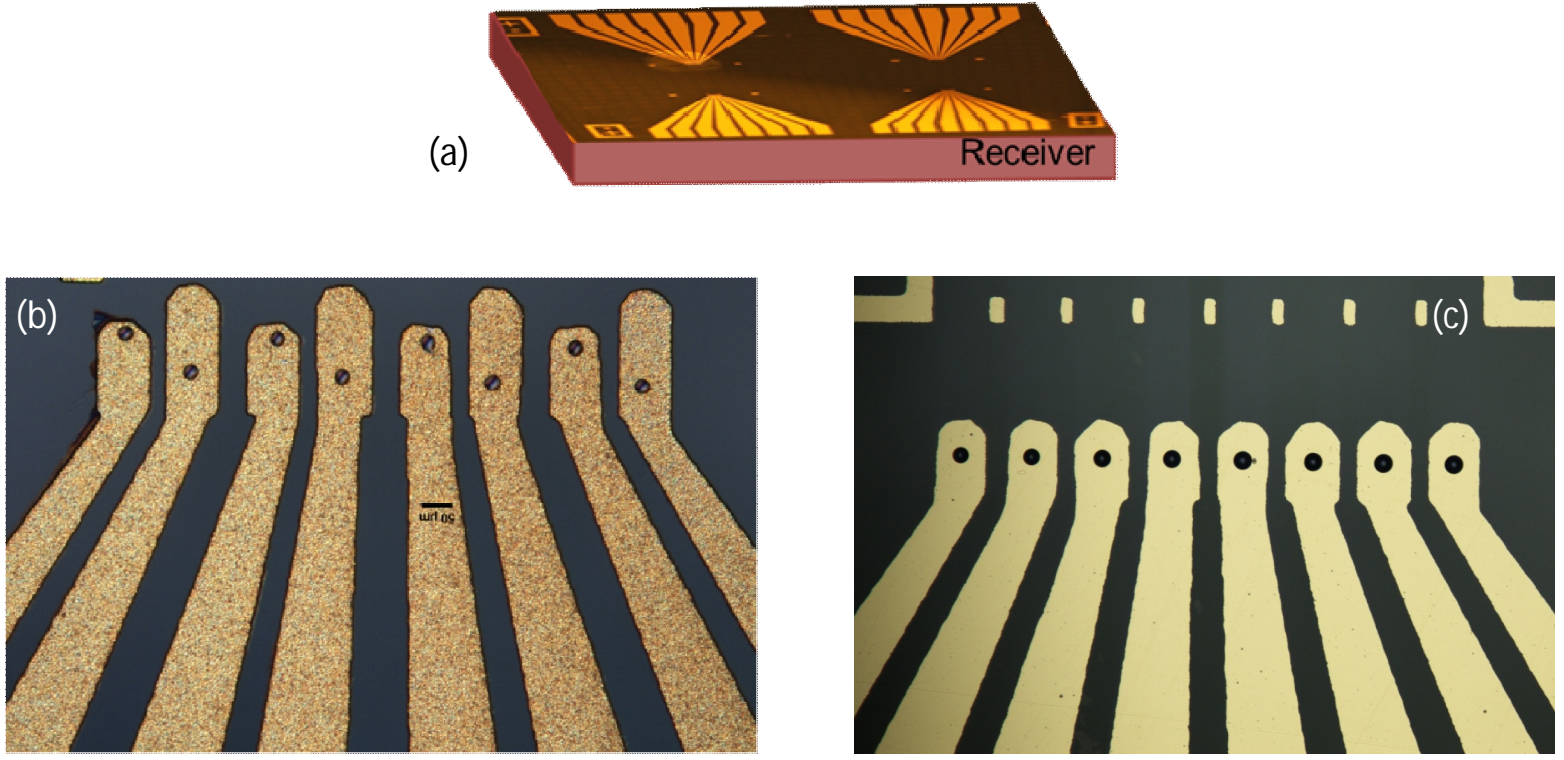

Fig. 1: (a) Illustration of the LIFT process; typical LIFT-assisted bumped receiver substrate for (b) PD and (c) VCSEL chip respectively.
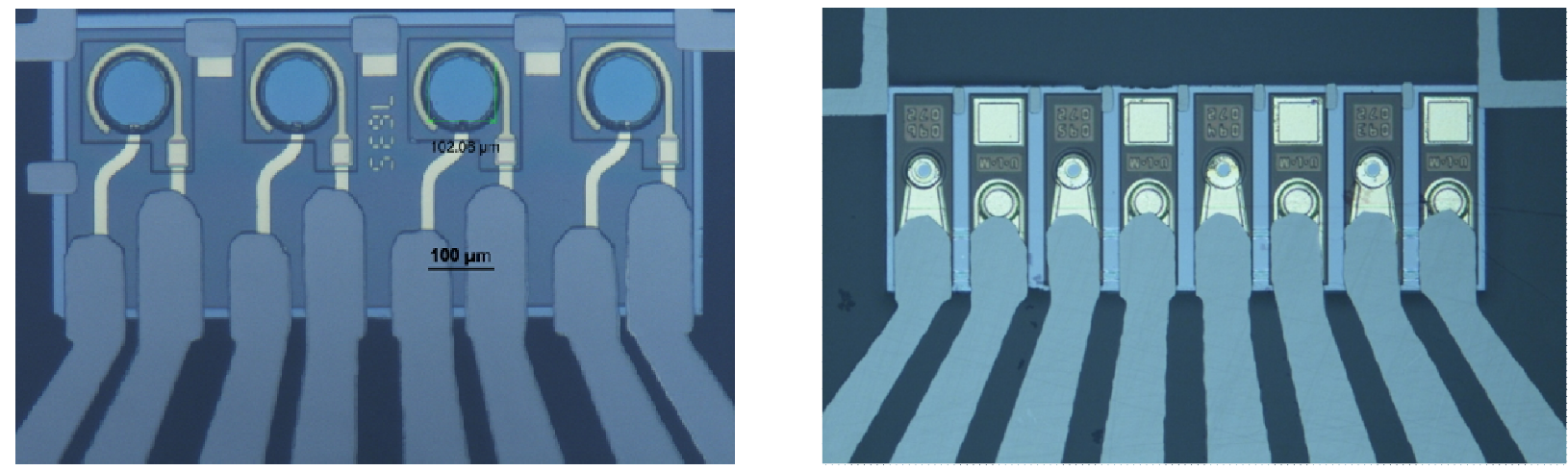

Fig. 2: Optical micrograph of (left) a PD and a (right) VCSEL chip FCB to a bumped receiver substrate as viewed from the back-side of the substrate. 


\subsection{Characterization of the FCB assemblies}

The FCB assemblies were then subsequently characterized both electrically and optically. Figure 3 (a) and 3 (b) show the typical IV and LIV curves recorded for the bonded PDs and VCSELs respectively. A $980 \mathrm{~nm}$ LED source was used for illuminating and characterizing the PDs. It should be mentioned that it was difficult to directly access the active area of the PDs with a fiber due to the glass substrate (flip-chip configuration). So the recorded photocurrent values (in $\mathrm{nA}$ ) are just representative results indicating the functioning of the PDs and not the actual photocurrent that is generated.

The curves clearly verify the successful functioning of the components post-bonding. To access the mechanical reliability of the bonded chips a die shear test was conducted using Dage 4000 series machine. The tests resulted in breaking the chips without detaching them from the substrates.

Table: Describing the key LIFT and FC bonding parameters

\begin{tabular}{|c|c|c|c|c|c|}
\hline $\begin{array}{c}\text { Donor } \\
\text { material }\end{array}$ & $\begin{array}{c}\text { Donor } \\
\text { thicknes } \\
\mathbf{s}(\boldsymbol{\mu m})\end{array}$ & $\begin{array}{c}\text { Donor- } \\
\text { receiver } \\
\text { separation }\end{array}$ & Fluence & $\begin{array}{c}\text { Bump } \\
\text { dimensions } \\
\text { (dia, height })\end{array}$ & $\begin{array}{c}\text { Bonding pressure, } \\
\text { temp. } \\
\left.\text { (gf, }{ }^{\boldsymbol{0}} \mathbf{C}\right)\end{array}$ \\
\hline Indium & $\sim 0.250$ & in contact & $270 \mathrm{~mJ} / \mathrm{cm}^{2}$ & $20 \mu \mathrm{m}, 1.5 \mu \mathrm{m}$ & 100,157 \\
\hline AgNP ink & $\sim 5$ & $50 \mu \mathrm{m}$ & $1.9 \mathrm{~J} / \mathrm{cm}^{2}$ & $20 \mu \mathrm{m}, 0.2 \mu \mathrm{m}$ & 100,150 \\
\hline AgNP paste & $\sim 5$ & $100 \mu \mathrm{m}$ & $550 \mathrm{~mJ} / \mathrm{cm}^{2}$ & $30 \mu \mathrm{m}, 2 \mu \mathrm{m}$ & 100,145 \\
\hline
\end{tabular}
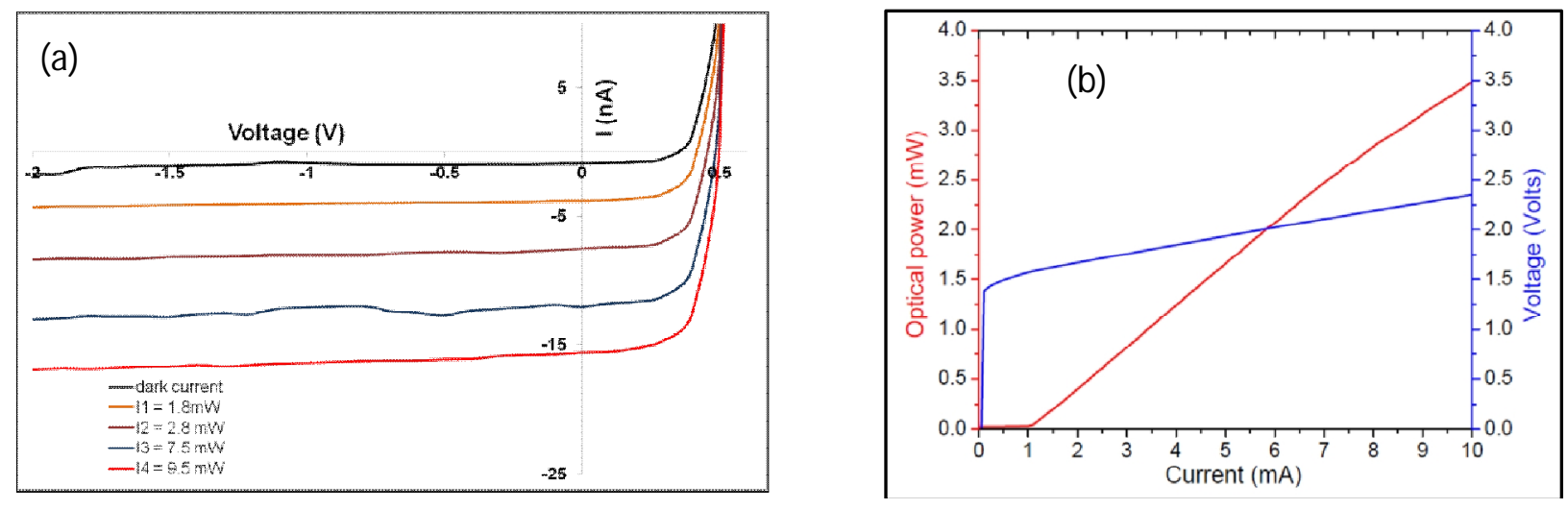

Fig. 3: Represents (a) IV curve recorded for a FCB PD and (b) LIV curve recorded from a FCB VCSEL assembly.

\section{Conclusions}

In conclusion, LIFT-assisted thermo-compression FC bonding and DC characterization of single VCSEL and PD chips was successfully demonstrated using indium and AgNP based ink and paste micro-bumps. Experiments to determine the life-time of the bonded devices and their RF characterization are currently underway. The presented results clearly show the capabilities of the LIFT technique for chip-level bumping and bonding in order to realize high-density FC interconnections.

\section{Acknowledgement}

Part of this work was carried out in the framework of the "MIRAGE" project, funded within the European Commission FP7 program.

\section{References}

[1] J.Bohandy et.al, "Metal deposition from a supported metal film using an excimer laser", J.Appl. Phys. 6 (4), 1538-1539 (1986).

[2] K.S.Kaur et.al,"Waveguide mode filters fabricated using laser-induced forward transfer", Opt. Exp., 19 (10), $9814-9819$ (2011).

[3] K.S.Kaur et.al, "Flip-chip bonding of vertical-cavity surface-emitting lasers using laser-induced forward transfer", APL, 104 (6), $061102-$ 061102-3 (2014). 